\title{
Special Issue: Supermodularity and Monotonicity in Economics
}

\author{
Rabah Amir ${ }^{1,2}$ \\ Published online: 12 October 2020 \\ ๑) Springer-Verlag GmbH Germany, part of Springer Nature 2020
}

This Special issue is composed of eight separate papers, each using the general methodology of supermodular games (such as Topkis's Theorem on monotone comparative statics, Topkis 1998) and/or monotone methods (such as Tarski's fixed point theorem). The topics of the papers lie in quite diversified areas of pure and applied economic theory, including the foundations of risk and uncertainty, principal-agent theory, Markov stopping games, recursive games, games with individually monotonic best responses, potential games, and oligopoly theory with and without network effects.

In what follows, we provide a brief introduction to each of the papers, in the order in which they appear in the Special Issue, along with some brief remarks on their respective related literature.

Gollier (2020) defines a notion of regret-risk aversion by requiring that the regretrisk-averse individual never choose the lottery that generates the larger risk of regret in a menu in which two lotteries have the same distribution of payoffs, with one generating a larger risk of regret in the sense of Rothschild-Stiglitz. It turns out that this notion is intimately connected to the concept of comparative concordance as introduced in economics by Epstein and Tanny (1980) and Tchen (1980); also see Amir and Lazzati (2016). The main result establishes that an individual is regret-risk-averse (-loving) if and only if her bivariate utility function is supermodular (submodular), i.e., has a positive (negative) cross partial derivative. We define regret-risk aversion in the small and in the large. We also show that regret-risk aversion tends to induce a bias in favor of the risky act in any one-risky-one-safe menu, in particular when the payoff of the risky choice is highly positively skewed. This is compatible with the "possibility effect" from prospect theory. Symmetrically, the aversion to rejoicing-risk can prevail when ex post utility is sensitive to the forgone worst payoff. Rejoicing-risk-seeking is compatible with the "certainty effect". Finally regret-risk-averse and rejoicing-riskseeking people behave as if they had rank-dependent utility with an inverse-S shaped

\footnotetext{
Rabah Amir

rabah-amir@uiowa.edu

1 Department of Economics, University of Iowa, Iowa City, IA 52242, USA

2 IMERA-AMSE, 13004 Marseille, France
} 
probability weighting function that reproduces estimates existing in the literature (e.g., Werner and Zank 2019; Lleras et al. 2019).

Laussel and Resende (2020) investigate how asymmetric information on final demand affects strategic interaction between a downstream monopolist and a number of upstream monopolists, who independently produce complementary inputs. This is modeled as an intrinsic private common agency game in which each supplier independently proposes a pricing schedule contract to the assembler, specifying the supplier's payment as a function of the assembler's purchase of its input. The authors derive a full characterization of equilibrium. While multiple equilibria exist, there is a unique Pareto-undominated Nash equilibrium from the suppliers' point of view. In this equilibrium, there are unavoidable efficiency losses due to excessively low sales of the good. However, suppliers may be able to limit these distortions by implicitly coordinating on an equilibrium with a rigid (positive) output in bad demand circumstances. In addition, bunching at the bottom arises when ex ante uncertainty is small enough, allowing suppliers to partially restore efficiency. In the limit, when uncertainty vanishes, the equilibrium output level tends toward the constant output level that maximizes aggregate suppliers' profits.

Boyarchenko (2020) considers a class of stopping games with two novel features. Unlike stochastic games with supermodular stage games, here the latter are affected by other players only via rates of information arrival. Unlike standard experimentation models with Poisson bandits (e.g. Das et al. 2020), the news arrival rate is hump or inverse U-shaped over time. In contrast to Poisson bandits, "humped" bandits are suitable for studying long-term negative effects of experimentation such as, e.g., harmful pollutants or drugs that have a potential for accumulation and long term storage in a human body; or positive effects, such as accumulated effort of economic agents or resources. The paper uses insights from the theory of supermodular games to establish existence and uniqueness of an equilibrium for this class of stopping games. As applications, the paper characterizes equilibrium outcomes in two games of strategic experimentation. Thus the suggested model of experimentation is almost as tractable as popular models based on exponential distributions, and produces qualitatively new results that better match the data than exponential models. In particular, in a situation when experimentation with risky projects may result in costly failures, the model predicts that termination of the experiment before arrival of the first failure is an equilibrium for a generic set of parameters. This paper joins a literature on exploiting basic insights from supermodular games in dynamic games (Amir 1996; Curtat 1996; Balbus et al. 2019; Guimaraes et al. 2020, among others).

Lasso de la Vega and Volij (2020) model a match as a recursive zero-sum game (i.e., a stochastic game with nonzero payoffs only at absorbing states) with three possible outcomes: Player 1 wins, player 2 wins, or there is a draw (in which case the point game is repeated). Play proceeds by steps from state to state. In each state, players play a "point game" and transit to the next state according to probabilities jointly determined by their actions and the state. This is thus a zero-sum reformulation of the binary Markov games analyzed in Walker et al. (2011), wherein play may fail to terminate, with positive probability thus yielding a zero payoff to both players. Examples of matches include tennis serves, penalty shootouts in soccer, and chess matches, among many others. The authors show that a particular value of a draw can 
be attached to each state so that an easily computed stationary equilibrium exists in which players' strategies can be described as minimax behavior in the point games induced by these values. The proof uses Tarski's fixed-point theorem (see Tarski 1955) applied to a suitably defined value operator. The paper thus provides an interesting alternative perspective on binary Markov games as fully-fledged zero-sum recursive games.

Barthel and Hoffmann (2020) define a new class of games (said to be of mixed heterogeneity, or GMH) wherein each player best responds either increasingly or decreasingly to an increase in each individual opponent's actions, as opposed to the joint action choice of opponents, as in games of strategic heterogeneity GSH (Barthel and Hoffman 2019). Solution concepts for the latter class, which includes games of strategic complements as a special case, have been shown to possess very useful properties, such as the existence of highest and lowest serially undominated strategies, and the equivalence of the stability of equilibria and dominance solvability. The main result of this paper gives necessary and sufficient conditions for when a GMH can be transformed into GSH in such a way so that these properties are preserved, allowing us to draw the same strong conclusions about solution sets in games that are not originally GSH. This is achieved by reversing the orders on the actions spaces of a given subset of players. Our second main result shows, rather surprisingly, that under mild conditions on the underlying ordering of action spaces, the reversal of orders is the only way in which such a transformation can be achieved. Applications of the basic results of the paper to aggregative games, market games, and crime networks are given.

Ewerhart (2020) derives necessary conditions for a smooth n-player game to admit an ordinal potential. In the class of smooth non-cooperative games, exact potential games and weighted potential games are known to admit a convenient full characterization in terms of cross-derivatives (Monderer and Shapley 1996). However, no analogous characterization is known for ordinal potential games. First, any ordinal potential game must exhibit locally pairwise strategic complements or substitutes at any interior equilibrium. Second, in games with more than two players, a condition is obtained on a (modified) Jacobian matrix at any interior equilibrium. Taken together, these conditions are shown to correspond to a local analogue of the Monderer-Shapley condition for weighted potential games. We identify two classes of economic games for which our necessary conditions are also sufficient: The standard Tullock contest and Bertrand competition with linear demands for differentiated goods and quadratic cost functions.

Cornand and Dos Santos Ferreira (2020) propose a unified setting nesting team and competition issues in price and quantity games, played by producers of either substitutes or complements with incomplete and dispersed information, to assess the social value of private and public information and compare the equilibria and social welfare of the two games. Without full cooperation, the competition motive introduces a strategy distortion and, with dispersed information, an informational distortion, both increasing in the intensity of competition. The former affects the response to the expected value of the fundamental, and the latter yields an inefficiently low (high) weight on public information under strategic complementarity (substitutability). Contrary to the latter, which vanishes in the absence of the competition motive, the former is eliminated, under strategic complementarity and dispersed information, at some positive strength 
of the competition motive, decreasing in information quality. This disparity creates a trade-off between the minimization of each distortion. As to the social value of public information, it is always positive, while that of private information may be negative under strategic complementarity, if competition is intense and the quality of private information relatively poor. Finally, it is more profitable to play under strategic substitutes, except possibly for an middle range of the intensity of competition if the quality of private information is again relatively poor. For related work, see e.g. Podhorsky (2020).

Gama et al. (2020) considers a general class of symmetric Cournot oligopolies with firm-specific network effects and investigates the effects of exogenous entry on market performance, and the profitability and welfare effects of horizontal mergers. Building on Amir et al. (2019) and Amir and Lambson (2000), the authors argue that firm-specific network effects are more widespread than one might think when considering indirect effects and fashion and other demand interdependencies. With strategic substitutes in the Cournot part of the model, per-firm output is declining in the number of firms, but industry output, price, per-firm profit, consumer surplus and social welfare may go either way in response to entry. Respective minimal sufficient conditions for each possibility are identified. The counter-intuitive conclusions tend to require strong network effects. The authors study the scope for profitability of mergers and the associated welfare effects. In a general analysis, the authors provide a sufficient condition on inverse demand for a merger to be profitable, which amount to requiring strong network effects. Under the condition that leads to higher industry output with entry, mergers are always social welfare-enhancing. Finally, the results of the paper are illustrated via two natural examples with linear and hyperbolic inverse demands, and thus closed-form solutions allowing an accessible view of the results of the paper.

\section{References}

Amir, R.: Continuous stochastic games of capital accumulation with convex transitions. Games Econ. Behav. 15, 111-131 (1996)

Amir, R., Lambson, V.E.: On the effects of entry in Cournot markets. Rev. Econ. Stud. 67, 235-254 (2000)

Amir, R., Lazzati, N.: Endogenous information acquisition in Bayesian games with strategic complementarities. J. Econ. Theory 163, 684-698 (2016)

Amir, R., Evstigneev, I., Gama, A.: Oligopoly with network effects: firm-specific versus single network. Econ. Theory (2019). https://doi.org/10.1007/s00199-019-01229-0

Balbus, Ł., Dziewulski, P., Reffett, K., Woźny, Ł.: A qualitative theory of large games with strategic complementarities. Econ. Theory 67, 497-523 (2019). https://doi.org/10.1007/s00199-017-1075-7

Barthel, A., Hoffman, E.: Rationalizability and learning in games with strategic heterogeneity. Econ. Theory 67, 565-587 (2019). https://doi.org/10.1007/s00199-017-1092-6

Barthel, A., Hoffmann, E.: Characterizing monotone games. Econ. Theory (2020). https://doi.org/10.1007/ s00199-019-01242-3

Boyarchenko, S.: Super- and submodularity of stopping games with random observations. Econ. Theory (2020). https://doi.org/10.1007/s00199-019-01198-4

Cornand, C., Dos Santos Ferreira, R.: The social value of information and the competition motive: price versus quantity games. Econ. Theory (2020). https://doi.org/10.1007/s00199-019-01179-7

Curtat, L.: Markov equilibria of stochastic games with complementarities. Games Econ. Behav. 17, 177-199 (1996)

Das, K., Klein, N., Schmid, K.: Strategic experimentation with asymmetric players. Econ. Theory 69, $1147-1175$ (2020) 
Epstein, L., Tanny, S.: Increasing generalized correlation: a definition and some economic consequences. Can. J. Econ. 13, 16-34 (1980)

Ewerhart, C.: Ordinal potentials in smooth games. Econ. Theory (2020). https://doi.org/10.1007/s00199-0 20-01257-1

Gama, A., Lahmandi-Ayed, R., Pereira, A.-E.: Entry and mergers in oligopoly with firm-specific network effects. Econ. Theory (2020). https://doi.org/10.1007/s00199-020-01316-7

Gollier, C.: Aversion to risk of regret and preference for positively skewed risks. Econ. Theory (2020). https://doi.org/10.1007/s00199-018-1154-4

Guimaraes, B., Machado, C., Pereira, A.-E.: Dynamic coordination with timing frictions: theory and applications. J. Public Econ. Theory (2020). https://doi.org/10.1111/jpet.12427

Lasso de la Vega, C., Volij, O.: The value of a draw. Econ. Theory (2020). https://doi.org/10.1007/s00199018-1140-x

Laussel, D., Resende, J.: Complementary monopolies with asymmetric information. Econ. Theory (2020). https://doi.org/10.1007/s00199-019-01197-5

Lleras, J.S., Piermont, E., Svoboda, R.: Asymmetric gain-loss reference dependence and attitudes toward uncertainty. Econ. Theory 68, 669-699 (2019). https://doi.org/10.1007/s00199-018-1138-4

Monderer, D., Shapley, L.S.: Potential games. Games Econ. Behav. 14(1), 124-143 (1996)

Podhorsky, A.: Environmental certification programs: How does information provision compare with taxation? J. Public Econ. Theory (2020). https://doi.org/10.1111/jpet.12450

Tarski, A.: A lattice-theoretical fixpoint theorem and its applications. Pac. J. Math. 5, 285-319 (1955)

Tchen, A.: Inequalities for distributions with given marginals. Ann. Prob. 8, 814-827 (1980)

Topkis, D.: Supermodularity and Complementarity. Princeton University Press, Princeton (1998)

Walker, M., Wooders, J., Amir, R.: Equilibrium play in matches: binary Markov games. Games Econ. Behav. 71, 487-502 (2011)

Werner, K.M., Zank, H.: A revealed reference point for prospect theory. Econ. Theory 67, 731-773 (2019)

Publisher's Note Springer Nature remains neutral with regard to jurisdictional claims in published maps and institutional affiliations. 